\title{
Gender and culture-related barriers to access to mother and infant health services in the Far North of Cameroon
}

DOI: http://doi.org/10.26758/8.1.11

Anita Nudelman (1), Deli Tize Teri (2) and Yafflo Ouattara (3)

(1) Ben Gurion University, Israel

(2) University of Yaounde I, Cameroon

(3) Joint United Nations Programme on HIV and AIDS (UNAIDS)

Address correspondence to: Anita Nudelman, Ben Gurion University, Israel, e-mail: anitanudelman@gmail.com

\begin{abstract}
Objectives. The Far North region of Cameroon has one of the highest neonatal, child mortality and poverty rates in the country. The aim of this study was to understand the gender and culture-related barriers to women's utilization of RMNCH (reproductive, maternal, neonatal and child health), including HIV services.

Material and methods. A participatory qualitative Rapid Assessment Process (RAP) was used and team members included women from the communities involved.

Results. Diseases affecting infants are explained by causes ranging from bad nutrition to sorcery and angry ancestors' spirits and traditional treatments are often preferred over medical services. Pregnancy is considered a natural state and a "women's issue", which is accompanied by cultural and religious norms that protect mother and fetus. Many women give birth at home with traditional birth attendants, who are trusted and follow accepted practices. Pregnant women living with HIV fear disclosing their status since this may lead to stigma and discrimination. Unequal gender power relations - including financial dependency on the husband - pose an additional barrier to service uptake, further enhanced by low male involvement in pregnancy-related health services. Lack of respect and confidentiality by health providers, associated with low-level availability and accessibility of services may also discourage their uptake.

Conclusions. The implementation of the RAP findings may enable innovative ways to increase culture and gender sensitivity of RMNCH services, including prevention of vertical transmission of HIV, in communities in Far North of Cameroon.
\end{abstract}

Keywords: RMNCH; culture; gender inequality; HIV; barriers to health services

\section{Introduction}

In many countries, especially in the developing world, women and children do not utilize available and essential health services due to a myriad of gender and socio-cultural barriers (Grieg et al, 2008).

Cultural perceptions, traditions and gender norms are an integral part of communities' way of life. They shape and influence daily activities, including accepted behaviors related to diseases, pregnancy, and childbirth as well as the selection of health care providers and treatment (Lori and Boyle, 2011). In some societies, unequal gender power relations reflect accepted gender roles that, 
combined with economic, political, and social factors, may pose serious barriers to access infant, child and maternal health services, including HIV related ones. For people living with HIV and AIDS (PLWHA), cultural barriers are further enhanced by the fear of stigma due to HIV disclosure and concomitant lack of support by partners, family, and community (Elwell, 2016; Gourlay et al, 2013).

Additional barriers to health services may arise from patient and provider's different cultural backgrounds, socio-economic and educational levels, and disrespectful attitudes and lack of confidentiality of some health providers (Karamagi et al, 2006). Among women living with HIV, these barriers are increased by the fear being stigmatized and discriminated against, thus deterring them from accessing specific health services (Painter et al, 2004; Reis et al, 2005). Furthermore, access to health services may also be influenced by their availability and accessibility, as well as by the quality of services available.

Maternal and infant mortality remain highest in developing countries due to barriers to utilization of health care (Cheptum et al, 2014), having hindered the Millenium Development Goals 4, 5 and 6, as well as mother to child transmission of HIV (United Nations, 2016).

This study was initiated by UNAIDS for the Sida/H4+ project (WHO, 2015) to accelerate the reduction of maternal, neonatal and infant mortality, as well as eliminating vertical transmission of HIV and reducing AIDS-related maternal deaths by half (UNAIDS, 2011). It focuses on the gender and culture related barriers to uptake of Maternal and Child Health services in the Far North Province of Cameroon, which holds a population of 3.8 million (approximately $18 \%$ of the national population), encompassing more than 50 different ethnic groups and religions such as Christianity, Islam, and Animism. The local economy is agro-pastoral, based in sustenance farming, with an incipient tourism industry which has decreased since 2014, due to the Boko Haram insurgency spilling into Cameroon from neighboring Nigeria (Rackley, 2017).

The study focuses on five-high need health districts in the Far North region: Maroua rural, Maroua urban, Guidigis, Moulvoudaye and Koza. These districts were selected due to their low coverage in health interventions, high proportion of home deliveries, low contraceptive prevalence rate, high neonatal and child mortality rates, and the highest prevalence of poverty in Cameroon (INS, 2011).

Maternal, infant and child health services are highly underused in this region, not only due to objective reasons but because they are often not gender and culture friendly (Bonono and OngoloZogo, 2012). Thus, the exploration of culture and gender barriers to services could contribute to a more context-sensitive healthcare enhancement, as well as to the decrease of maternal and infant morbidity and mortality, including prevention of mother-to-child transmission (PMTCT).

\section{Material and methods}

The Rapid Assessment Process (RAP) was the methodological approach chosen to understand the gender and culture related barriers to women's use of maternal and child services (including HIV ones) in three representatives sites within the selected health districts in the Far North region of Cameroon: Maroua (both urban and rural), Guidiguis and Koza. This method had previously been used in a similar study initiated by UNAIDS in specific sites in five high burden countries, most of them in Africa (Nudelman, 2013). The Rapid Assessment Process is an intensive team-based qualitative technique to quickly develop an understanding of a situation from the insiders' perspective (Beebe, 2004). Instead of extended fieldwork, it is based on intensive and participatory team interaction and multiple cycles of data collection followed by analysis (Scrimshaw et al, 1991). Therefore, the team trained for the project was entirely composed of 
members native to the Far North, and although some did not currently reside in the region, they had close ties to the communities assessed since they had all grown up there and returned frequently to see their families. The team included community women from the different sites whose insight and participation proved to be invaluable.

The ethnicity of the participants - including key informants and Focus Group Discussion members - varied across the RAP sites. In urban Maroua, the RAP included participants from heterogonous ethnic groups such as Peul, Kapsiki, Mousgoum, Guizigua, Kanouri and Moufou. In rural Maroua, the ethnicity of most participants was Guizigua and Moufou. In Guidiguis, participants were Toupouri and Massa, while the participants in Koza belonged to the Mafa ethnic group (Boutrais 1984).

The study focused on the barriers to services in selected sites and not on an in-depth study of specific ethnic groups since access to services was similar across them.

The purpose of the Rapid Assessment Process was to explore the barriers from women's and communities' perspectives in order to promote gender and culture-sensitive health services. Fieldwork was conducted in April 2014, encompassing 132 participants who were interviewed either in-depth or in focus group discussions. It included key informants (community leaders and health providers), pregnant women, mothers of small children (some living with HIV), male partners as well as other men from the community. The interview guides were culturally adapted by the research team and translated into Fulfulde, the local language in most of the rapid assessment sites. An additional 18 community members were interviewed informally.

\section{Results}

\section{Barriers to the use of maternal and infant health services, including PMTCT}

The access and utilization of health services were influenced by different barriers, which resulted from a combination of factors, such as poverty, a weak health system and socio-cultural barriers, including fear of stigma and discrimination related to HIV. In addition, certain traditional cultural perceptions may influence a woman's decision to attend a health facility for an antenatal visit or for childbirth (i.e. considering that it is natural to deliver at home), while gender also plays an important role in some of the aforesaid factors. For example, women may not disclose their HIV status for fear of abandonment, loss of economic support, emotional and physical abuse, and discrimination (Medley et al., 2004). The findings will be presented in 5 categories: (1) Barriers related to socio-cultural perceptions; (2) Barriers related to gender roles and low male involvement; (3) Barriers related to perceptions of health services and providers; (4) Fear of disclosure, stigma, and discrimination and (5) Lack of resources, poor infrastructure, and poverty

\section{Barriers related to socio-cultural perceptions Barriers related to the perception of diseases}

The rapid assessment's findings indicate that wide-spread diseases affecting infant morbidity and mortality are often explained by causes that range from bad nutrition to sorcery. In general, treatments for these diseases in the different communities range from auto-medication to traditional practices, according to the perceived illness causation, with official health services as the last option.

Some diseases, such as tandaw (a type of mycoses) and bedel (a stomach disease), are believed to be caused by angry ancestors' spirits due to lack of sacrifices in their honor nowadays: 
"Before, we used to sacrifice cocks (roosters) next to the water source in the mountain so that it would not dry up; or we cracked eggs instead. But, for a few years already, this rite has not been kept." (Male FGD, rural Maroua)

Therefore, it is believed that if children suffering from bedel go to a hospital, the disease may get worse and even cause death, since according to the community's perceptions, bedel does not like injections or white man's treatment and only requires sacrifices or scarification.

Among some of the communities assessed, malaria is believed to be caused by eating raw vegetables, millet stems, and sour milk:

"At the beginning of harvest season, the children here eat many millet stems. There is a type of wild millet which has a sweet stem-like sugar cane, called "tchibe" or "lewlewa" amomg the Kapsiki. Parents cultivate them and often sell them in the village or the market. Among us, the harvest season of these stems is always accompanied by malaria epidemics." (Woman, urban Maroua)

Considering the aforesaid, malaria is often perceived as a regular seasonal disease that can be treated and will eventually pass, and thus it is not considered necessary to access a health facility:

"In this village, there are traditional healers who are very efficient; if you have simple malaria, they give you a medicine and you are cured." (Male FGD, rural Maroua)

Although many participants are aware that malaria is transmitted by mosquitoes, nets may not be used due to the belief that they are sprayed with toxic materials or that they are distributed by the white man to kill black people, a belief which is also common to some perceptions of the origin of HIV in Africa (Rodlach, 2006).

"What is given free of charge, is surely harmful (so beware and do not use it)." (Woman living with HIV, Maroua)

Knowledge and perceptions of HIV/AIDS vary across the different sites, and this disease was practically unknown in some remote rural areas. Nevertheless, local names for the disease both reflect and influence cultural perceptions of HIV. For example, it is often referred to as "plastique", which is also an incurable disease that kills animals (through slow waste and diarrhea) that is believed to be caused by eating plastic or "leda". In addition, beliefs about HIV's origins and modes of transmission, such as being caused by sorcery or a punishment from God, tend to reinforce fears and stigma, while posing barriers to service access:

"The disease is the consequence of the sins and the sign of the end of the world. In fact, diseases and disasters are an announcement or the signal of Islam's prophecy. Allah will not change as long as the community does not change its ways." (Imam, Maroua)

These beliefs also pose barriers to attending an antenatal consultation or "kilo", which participants usually equal to testing for HIV since it is a standard practice to test all women during antenatal consultations (ANC).

Considering the widespread perceptions and beliefs related to HIV, culturally-appropriate treatments are often preferred over the modern anti-retroviral one and may include Christian and Muslim prayers, as well as the use of traditional medicine. 
For some Christian revival Churches, the treatment is through prayers. Thus, the faithful can obtain a cure to HIV through their belief and confidence in God:

"I know two sisters in Christ who were found to be HIV positive. They belong to our Church. The Church's faithful fasted and prayed for them for days. After that, they were tested again three times, and at the end the result became negative." (Female FGD, urban Maroua)

\section{Barriers related to perceptions of pregnant women and childbirth}

Pregnancy is viewed as a natural state, a "benign disease", and considered a women's issue. Many cultural and religious norms must be kept during pregnancy in order to protect the mother and the fetus from dangers, mostly concerning nutrition and behavioral taboos. Such practices do not necessitate the use of health services:

"A pregnant woman should not eat large lizards (varanus), locusts and certain other animals such as rabbits. According to the tradition, the newborn child can look like the animal the mother ate or it will make the child very thin." (Woman, Guidiguis)

Regarding pregnant women living with HIV, some of them felt ashamed and feared to disclose their status since they may be considered immoral and irresponsible by community members because their babies will inevitably be born HIV positive and will probably die soon after birth:

"A pregnant woman who is seropositive is a criminal because her days are numbered. She will die and abandon her baby." (Man, rural Maroua)

In addition, women living with HIV are often rejected and even isolated, especially in polygamist families:

"People keep away from me. When I prepare food, the neighbors and even my brothers tell their children not to eat my food." (Woman living with HIV, Guidiguis)

Modern family planning is not a developed maternal service in the region, with the exception - to a limited extent - of Maroua city. In a society where children (especially boys) are highly valued and considered a sign of richness, it is usually the man's prerogative to decide on this issue. Nevertheless, traditional ways of spacing children exist, involving "marabous" (traditional healers) and some traditional birth attendants:

"I can hinder a woman from (getting pregnant) giving birth for some years, but it depends on couple agreeing to this. If a woman comes to see me with her husband to space out their children, I will do it! '”(Traditional birth attendant, Koza)

\section{Gender roles and low male involvement}

In line with culturally accepted gender role norms and expectations, a woman is expected to be subservient, and socially and financially dependent on her husband and in-laws. Thus, unequal gender power relations can pose a significant barrier to maternal health services, considering that the husband is traditionally the main decision-maker in the family and his consent is required for a woman to access them. Men may oppose antenatal visits due to a combination of factors, such as 
lack of gender and culture-appropriate treatment at a health facility, where male nurses treat Muslim women (and may also see them naked):

"Yes some Muslims do not accept that the men (male nurses) handle their wives. But it is not the religion or the Islam which forbids, it is the men themselves who are against it. In order to solve this problem, we have created a maternity clinic here in Bamaré quarter and we have female nurses." (Religious authority, Maroua)

These barriers can be further reinforced by lack of trust in the efficacy of the services, as well as the unwillingness to spend money on transport, which is a husband's prerogative in the patriarchal family structure in this region:

"...for example, I asked my husband for money, he refused. I wanted to trade (in order to get money for transport) he also refused. So with what can I go to the antenatal consultation?" (Pregnant woman, urban Maroua)

If a woman disobeys her husband, she may be at risk of physical or emotional abuse and even abandonment:

"One night, I was beaten by my husband because I had suggested going to the kilo (antenatal consultation). As my husband knew that I would then ask him for money for the kilo, he began to beat me." (Female FGD, rural Maroua)

HIV was also found to have a gender connotation, since it is considered a female disease, mostly transmitted by women:

"Women refuse to get tested here in the Far North because they are afraid to find out their status. Women are often accused of being the vector that transmits the disease. Therefore, if a woman decides to be tested, she will be afraid of stigma. If she finds out that she is seropositive, she is forced to hide her status to avoid being divorced." (Woman living with HIV, urban Maroua)

Male partners' limited participation in issues related to pregnancy and childbirth, both at home and during women's visits to health facilities, poses an additional barrier that may discourage service uptake - including PMTCT. This is strengthened by the widespread perception that issues related to pregnancy and childbirth are "women's business" (which is an accepted gender role). Furthermore, some men refuse to get involved due to fear of social embarrassment, since a man who accompanies his wife may be viewed in a negative way by the community and considered "a man dominated by a woman". Thus, men often discourage maternal and HIV service uptake or do not provide their wives with the necessary emotional and logistical support.

A different view was expressed by a health provider in urban Maroua, who suggested that if men got involved, maternal health service utilization might increase:

"Men should become aware that the hospital is a good thing, otherwise the women will not change. It is the man who decides and if he asks his wife do some thing or other, she will do it (related to pregnancy, etc.). If men join women, things will work out”. 


\section{Barriers related to perceptions of health services and providers}

The adherence to cultural norms and beliefs that may conflict with medical treatment is sometimes reinforced by mistrust of health providers, further discouraging visits to healthcare facilities. Thus, women often prefer to continue with traditional birth practices:

"Women are used to delivering their babies well at home; so, the community does not see the importance of going to a hospital." (Health provider, Koza)

"I never go to the hospital for delivery. I do it like going to the toilet (squatting) and I deliver. Why is it important to go to the hospital?" (Woman, Guidiguis)

"A long time ago, a 'Mitsekar' Mafa clan ancestor declared that the (clan's) daughters must give birth at home; following the death of a pregnant woman due to exhaustion on the long way to give birth in the hospital. Since his message, some families continue to give birth at home to avoid a curse (from the ancestors) to befall their families." (Female FGD Koza)

Although sometimes antenatal services were encouraged by providing incentives and small gifts to women, they are not consistently used, except in case of emergencies. Even women who attend antenatal consultations tend to give birth at home, assisted by traditional birth attendants (TBA), who are familiar and well-respected members of the communities, follow socio-cultural norms, and are trusted.

Health services in some rural areas are almost inexistent or can be very distant. In urban areas, many women prefer private facilities to government ones because of the quality of services and the friendlier attitudes of the health providers:

"At the central hospital, there are no chairs for us to sit on! The women are (waiting) in the sun and nurses do not even look at you!'” (Woman, urban Maroua)

This is further compounded by previous experiences of being badly treated while accessing modern maternal services. Some pregnant women indicated that they were not treated respectfully or were often insulted, increasing the barriers to services:

"Nurses mock women here in Koza. They often say: 'we are going to operate a bull' to describe a pregnant woman who has a big body or 'we are going to operate a goat' to describe a pregnant woman with complications." (Traditional village chief, Koza)

"Male nurses sometimes laugh (mock) at the nudity of the women who come to give birth in the hospital. After the delivery, they stay to discuss and to describe what her genitalia looks like. The professional secrecy is not guaranteed! They report everything they see in the village. That is why some women do not go to the hospital." (Woman, Guidiguis)

Thus, negative attitudes furthered by lack of respect and confidentiality by certain health providers - mainly in public health facilities - pose a major barrier to maternal services in some communities. Naturally, women turn to traditional birth attendants, who are more familiar and supportive.

Although antenatal and natal services have a low utilization rate in the Far North Region, postnatal ones are accessed more frequently. These services enable mothers to obtain vaccination 
carnets, as well as birth certificates for their babies (necessary for benefits and bureaucratic issues, such as registration for school).

\section{Fear of disclosure, stigma, and discrimination}

Women usually become aware of their HIV status when they attend antenatal consultations or give birth since testing for HIV is a standard procedure. The test results are sometimes disclosed without counseling or in insensitive ways, by informing a woman: "You have AIDS", which can further increase her fears and distress.

Social stigma is the main cause due to which participants living with HIV failed to disclose their status to their partners, families and other community members. Women living with HIV generally faced more barriers to treatment than males, due to cultural perceptions of gender roles that tolerate male, but not female infidelity:

"If a man gets infected with AIDS, the community considers he did it because he succumbed to temptation. But if it is a woman, it is a sin because she does not have the right to do things out of wedlock." (Religious Authority, urban Maroua)

The fear of gossip and disclosure is particularly difficult for women, who may fear violence or abandonment by their partners if their HIV status is revealed:

"A woman is much more exposed (than men) to the whispers (gossip) of the people around her." (Woman, urban Maroua)

This barrier is further enhanced since pregnant women living with HIV-as well as their male partners - fear that their status may be revealed if they are seen attending a specific health facility that is associated with HIV. For instance, in some health districts, maternal services are exposed to the public, so women who come to be tested for HIV are received in groups in open spaces, without respecting their privacy:

"When we pass on the road, we see the women under the shed in the hospital. The women don't want people to know that they came for the "kilo" (testing) in a place like an ANC or a health center that symbolize HIV positive." (Woman, Koza)

Women living with HIV are sometimes also stigmatized by health providers. A few were offensive towards people living with HIV who came to collect their antiretroviral medicines:

"Because patients get impatient while waiting for services, she (a nurse) told us in a loud voice: 'it is not me who sent you to get AIDS: That shamed us and discouraged others to come and get their medicine. Fortunately, she was chased away from the hospital." (Woman. urban Maroua)

Thus, negative attitudes and the breaches of confidentiality by some health providers can pose a strong barrier for women living with HIV to attend health facilities. Furthermore, the distribution of antiretroviral medicines often tends to be irregular, with people living with HIV being shuffled from one health center to another. This results in a loss of time and resources while increasing the visibility of women living with HIV (and concurrently their stigmatization), as well as their negative perception and their utilization of HIV services. 
In many communities, there was little information about vertical transmission of HIV or PMTCT treatment, with most exceptions in Maroua city.

\section{Lack of resources, poor infrastructure, and poverty}

Participants' financial constraints pose a barrier to access maternal and HIV health services and are also associated with the low level of services available and the shortage of trained personnel and medicines. Some women complained of visits to undersized, underequipped, and understaffed health facilities, in which services were not adequate. In certain remote areas, the complete lack of availability of services led to increased difficulties in travel and additional expenses:

"The reason people refuse to go to the hospital is the lack of financial means ... the high cost of the antenatal consultation limits their attendance, since here women are not emancipated enough to create jobs and provide for their own needs. " (Female FGD, woman, urban Maroua)

For women in some villages in rural areas, the distance to the nearest basic health facility ranged from $15 \mathrm{~km}$ to $35 \mathrm{~km}$, posing an objective barrier that is further enhanced by poverty (estimated at $41 \%$ of the population in this region):

"Here in Ngarmace, we are $10 \mathrm{~km}$ away from Torock, $24 \mathrm{~km}$ from Guereme and $19 \mathrm{~km}$ from Guidiguis. Do you realize that a pregnant woman risks giving birth on the way! So why not use a traditional midwife (instead)?" (Pregnant woman, Guidiguis)

When considering HIV health services, availability, and accessibility is often critical for testing and treatment, including PMTCT:

"(HIV) tests are not always available... And when we got the tests they were already outdated. When the woman you advised to do the screening finds that the test is not available, it becomes difficult for her to come back tomorrow and she will not come... and many women who attend ANC remain in the village without being tested." (Health provider, Koza)

Some participants recalled better times, when mobile services outreached their distant communities and they did not have to walk or to travel long distances:

"In the past, there were doctors who came to do the kilo (antenatal consultation) for the women here in the village. They used to do it in my house; but for a long time now, they are not coming anymore." (Village chief, rural Maroua)

In addition, people are often faced with the dilemma of having to choose between allocating funds for basic food or for covering travel expenses to a health facility, which can result in an additional barrier to service utilization.

"Instead of spending more than $10000 \mathrm{~F}$ in the hospital, we can give birth for 1500 or $2000 \mathrm{~F}$. The rest of money can help us to feed them." (Woman, Koza) 


\section{Discussions}

Cultural perceptions, beliefs, and practices, as well as gender inequality, still pose a challenge to child, maternal, and HIV services in many African countries (Cheptum, 2014), notwithstanding the advances made by Millennium Development Goals (United Nations, 2016). These are further compounded by socio-economic factors and others related to health facilities factors, posing significant barriers to service utilization.

In the Far North of Cameroon, some diseases that affect children are considered seasonal or related to supernatural causes and are treated at home or by traditional healers, sometimes delaying essential medical treatment, as in the case of malaria (Makundi et al, 2006).

Pregnancy and childbirth are often managed at home (USAID and MCHIP, 2012) since they are considered natural processes of the life cycle. Thus, traditional birth attendants are often preferred since they are respected members of the community, share cultural norms and beliefs, and are considered more supportive than providers in health facilities (Lori and Boyle, 2011; Kaombo, 2013). In addition, beliefs related to HIV (Dickinson, 2013), such as it being caused by witchcraft or divine punishment, are still widespread (Rodlach, 2006). Considering the aforesaid, access to maternal health services in general, as well as to specific HIV ones, such as prevention of vertical transmission, may often be discouraged (USAID and MCHIP, 2012; Ganlel et al, 2015).

Gendered roles regarding maternal health are clear-cut (Godlove et al, 2010). However, although pregnancy and childbirth are perceived as women's responsibility, usually a woman must obtain her partner's consent in order to access maternal and HIV care, due to traditional social structures that reinforce gender inequality (Grieg et al, 2008). Considering that partner support is essential for antenatal consultations and especially for prevention of vertical transmission, low male participation continues to pose a serious barrier to the utilization of these health services (Gourlay et al, 2013).

Women living with HIV experience higher levels of stigma than men and may be blamed for bringing the disease into the family, as they are usually the first person in a family to find out their status when tested during ante-natal consultations. Many women fear disclosing their HIV status to their husbands since this may lead to violence, abandonment and divorce (One in nine Campaign, 2012), thus affecting their access to services (Moges, 2009). In addition, some women indicated that if they were seen at a facility with HIV services, community members would suspect them of living with HIV, which could lead to gossip and discrimination, thus further discouraging them from accessing services, including PMTCT.

Lack of trust of health services was attributed to a combination of factors. Health providers are often identified with modern medicine, which is not widely used in some communities that still foster traditional perceptions and beliefs. Consequently, misunderstandings, wariness, and mistrust are inevitable (Bohren et al, 2014). This can be enhanced by the negative attitudes and behavior of some health providers, as was noted by women living with HIV, who suffered from discriminatory treatment and breaches of confidentiality, thus increasing their fear of disclosure through service utilization (Famaroti, Fernandes and Chima, 2013; Karamagi et al, 2006).

In addition to the barriers mentioned, objective factors such as poverty, availability, and accessibility to the child, maternal, and HIV health services contribute to their low uptake in the region. Concurrently, the distance to these facilities may be aggravated by bad infrastructure, lack of transport, and the costs and time involved in traveling (Tize Teri, 2016), further affecting women's mobility, which is already limited by accepted cultural norms and gender power relations (Grieg et al, 2008). 


\section{Conclusions and recommendations}

The study highlighted the need for gender and culture transformative approaches to overcome barriers to maternal and child services in the Far North Region of Cameroon, including prevention of vertical transmission (Nudelman, 2013). Sensitization and awareness campaigns should target the main diseases that affect maternal and infant health. Issues related to pregnancy, childbirth and gender equality, as well as stigma and discrimination, may be better addressed through innovative techniques while encouraging active community participation. These may include: community theatre, peer training, activities in churches and football clubs, as well as interactive radio programs in local languages.

At the same time, ongoing health provider sensitization is essential to enhance culture and gender sensitive health care. Implementing a task shifting process (WHO, 2008) could reduce the overburden of health providers and extend health services to more remote areas. Thus, training of and collaboration with traditional birth attendants could have a significant impact on maternal health in the region (Kaombo, 2013), in addition to a more active involvement of community health liaisons (Nudelman and Tezi Teri, 2014).

Male engagement in maternal and HIV services should also be encouraged, while taking into account traditional gender and socio-cultural issues (Godlove et al, 2010). Finally, empowering women from the different communities in the region - including women living with HIV - through capacity building, could enable their participation in health care services, while fostering gender equality and contributing to stigma reduction and to health service uptake (Mothers-to-mothers Uganda, 2016). Considering the objective barriers mentioned such as accessibility, reinstatement of mobile clinics could bring the maternal and HIV services closer to remote communities.

The implementation of the findings and recommendations of this rapid assessment may contribute to the enhancement of health care in culture-specific contexts in the Far North province of Cameroon. Consequently, it may reduce morbidity and mortality, as well as eliminate new HIV infections among children in the region while also sustaining their mothers' health and well-being (UNAIDS, 2015).

\section{Bibliography}

1. Beebe, J., 2004. Rapid Assessment Process, Encyclopedia of Social Measurement, SOME00562, pp.1-7.

2. Bohren, M.A., Hunter, E.C., Munthe-Kaas, H., Souza, J.P., Vogel, J.P., and Gülmezoglu, A.M., 2014. Facilitators and barriers to facility-based delivery in low- and middle-income countries: a qualitative evidence synthesis. Reproductive Health, 11(1), pp.71. Available at: http://www.reproductive-health-journal.com/content/11/1/71 [Accessed 18 August 2017].

3. Bonono, R.C. and Ongolo-Zogo, P., 2012. Optimizing the use of antenatal care services in Cameroon. Centre for Development of Best Practices in Health-Central Hospital Yaoundé, Cameroon.

4. Boutrais, J., 1984. Le Nord du Cameroun: des hommes, une région. Paris: ORSTOM.

5. Cheptum, J., Gitonga, M., Mutua, E., Mukui, S., Ndambuki, J. and Koima, W., 2014. Barriers to Access and Utilization of Maternal and Infant Health Services in Migori, Kenya, Developing Country Studies 4 (15), pp.48-52.

6. Dickinson, D., 2013. Myths or theories? Alternative beliefs about HIV and AIDS in South African working class communities, African Journal of AIDS Research, 12(3), pp.121-130, DOI: 10.2989/16085906.2013.863212. 
7. Elwell, K., 2016. Facilitators and barriers to treatment adherence within PMTCT programs in Malawi. AIDS Care, 28(8), pp.971-975.

8. Famaroti, T.O., Fernandes, L., and Chima, S.C., 2013. Stigmatization of persons living with HIV/AIDS by healthcare workers at a tertiary hospital in Kwazulu-Natal, South Africa: a crosssectional descriptive study. BMC Medical Ethics, 14(Suppl. 1), pp.56. Available at: https://doi.org/10.11.86/1472-6939-14-51-56

9. Ganle1, K.J., Otupiri., E., Parker, M. and Fitzpatrick, R., 2015. Socio-cultural Barriers to Accessibility and Utilization of Maternal and Newborn Healthcare Services in Ghana after Userfee Abolition. International Journal of Maternal and Child Health, 3(1), pp.1-14.

10. Gourlay, A., Birdthistle, I., Mburu, G., Iorpenda, K. and Wringe, A., 2013. Barriers and facilitating factors to the uptake of antiretroviral drugs for prevention of mother-to-child transmission of HIV in sub-Saharan Africa: a systematic review. Journal of the International AIDS Society, 16(1):18588.

11. Grieg, A., Peacock, D., Jewkes R., and Msimang, S., 2008. Gender and AIDS: Time to Act. AIDS 22 (Suppl. 2 2), S35-S43. Available at: https://journals.1ww.com/aidsonline/fulltext/2008/08002/Gender_and_AIDS time_to_act.7.asp $\underline{x}$ [Accessed 10 July 2017].

12. Kaombo, A.J., 2013. Impact of training traditional birth attendants on maternal mortality and morbidity in Sub-Saharan African countries. Tanzania Journal of Health Research, 15(2), pp.134-142.

13. Karamagi, C.A.S., Tumwine, J.K., Tylleskar, T., and Heggenhougen, K., 2006. Antenatal HIV testing in rural eastern Uganda in 2003: incomplete rollout of the prevention of mother-to-child transmission of HIV programme, BMC International Health and Human Rights, 6(6), doi:10.1186/1472-698X-6-6.

14. Lori, J. R., and Boyle, J.S., 2011. Cultural Childbirth Practices, Beliefs, and Traditions in Postconflict Liberia. Health Care for Women International, 32(6), pp.454-473.

15. Makundi, E.A., Malebo, H.M., Mhame, P., Kitua, A.Y. and Warsame, M., 2006. Role of traditional healers in the management of severe malaria among children below five years of age: the case of Kilosa and Handeni Districts, Tanzania. Malaria Journal, 5:58. Available at: http://www.malariajournal.com/content/5/1/58 [Accessed 12 July 2017].

16. Medley, A., Garcia-Moreno C., McGill, S. and Maman, S., 2004. Rates, barriers and outcomes of HIV serostatus disclosure among women in developing countries: Implications for prevention of mother-to-child transmission programmes, Bulletin of the World Health Organization, 82(4), pp.299-307.

17. Moges, G., 2009. Factors Influencing Utilization of PMTCT Services in Addis Ababa, Ethiopia, $45^{\text {th }}$ International Course in Health Development, KIT (Royal Tropical Institute), Free University, Amsterdam. Available at: http://www.bibalex.org/search4dev/files/351971/185326.pdf [Accessed 15 July 2017].

18. Nkuoh, G.N., Meyer, D.J., Tih, P.M. and Nkfusai, J., 2010. Barriers to Men's Participation in Antenatal and Prevention of Mother-to-Child HIV Transmission Care in Cameroon, Africa. Journal of Midwifery \& Women's Health, 55(4), pp.363-369.

19. Nudelman, A., 2013. Gender-related barriers to services for preventing new HIV infections among children and keeping their mothers alive in high-burden countries. UNAIDS: Discussion Paper. Available at: http://www.unaids.org/en/media/unaids/contentassets/documents/unaidspublication/2013/20131 2_discussion-paper_Gender-HIV-services_PMTCT_en.pdf 
20. Nudelman, A. and Tize Teri, D., 2014. Evaluation des obstacles liés au Genre dans l'accès aux soins de santé maternelle et infantile y compris la PTME dans les districts de santé de Maroua urbain/rural, Koza, Moulvoudaye et Guidiguis à l'Extrême-Nord du Cameroun. Rapport prépare pur CASSRT et ONUSIDA.

21. Painter, T. M., Diaby, K.L., Matia, D.M., Lin, L. S., Sibailly, T.S., Kouassi, M.K., Ekpini, E.R., Roels, T.H., \& Wiktor, S.Z., 2004. Women's reasons for not participating in follow up visits before starting short course antiretroviral prophylaxis for prevention of mother to child transmission of HIV: qualitative interview study. BMJ,329(7465), pp.543. doi: $10.1136 / \mathrm{bmj} .329 .7465 .543$.

22. Rackley, E.B., 2017. Cameroon's Far North: Responding to Boko Haram Edward B. Rackley. Oxford Research Group Briefing. Available at: http://www.oxfordresearchgroup.org.uk/publications/briefing_papers_and_reports/cameroon\%E 2\%80\%99s_far_north_responding_boko_haram [Accessed 17 September 2017].

23. Reis, C., Heisler, M., Amowitz, L., Moreland, R.S., Mafeni, J.O., Anyamele, C. and Iacopino, V., 2005. Discriminatory Attitudes and Practices by Health Workers toward Patients with HIV/AIDS in Nigeria. PLoS Medicine, 2(8) e246.| www.plosmedicine.org 0752.

24. Rödlach, A., 2006. Witches, Westerners and HIV: AIDS and Cultures of Blame in Africa. Walnut Creek, CA: Left Coast Press.

25. Scrimshaw, S.C.M., Carballo, M., Ramosand, L. and Blair, B.A., 1991.The AIDS rapid anthropological assessment procedures: A tool for health education planning and evaluation. Health Education Quarterly, 18(1), pp.111-123.

26. Tize Teri, D., 2016. Le recours à l'accouchement à domicile à l'Extrême-Nord du Cameroun: une logique prédisposant aux soins de santé parallèles. Offres, recours et accès aux soins de santé parallèles en Afrique. Des acteurs en quête de légitimité sociale, médicale et institutionnelle. Éd. Différance Pérenne, pp.257-269.

27. *** Mothers-to Mothers, Uganda, 2016. Available at: https://www.m2m.org/where-wework/uganda/[Accessed 17 July 2017].

28. ***Institut National de la Statistique - INS, 2011. 4eme Enquête Démographique et de Santé et a indicateurs Multiples EDS-MICS4, Yaoundé, Cameroon.

29. ***One in nine Campaign, 2012. We were not meant to survive: Violence in the Lives of HIV Positive Women in South Africa. Available at: htpp://www.oneinnine.org [Accessed 10 August 2017].

30. ***UNAIDS, 2011. Countdown to zero: Global plan towards the elimination of new HIV infections among children by 2015 and keeping their mothers alive. Available at: http://www.unaids.org/en/media/unaids/contentassets/documents/unaidspublication/2011/20110 609 jc2137_global-plan-elimination-hiv-children_en.pdf [Accessed 15 June 2017].

31. ***UNAIDS, 2015. UNAIDS Strategy 2016-2021: On the Fast Track to end AIDS. Available at: http://www.unaids.org/en/resources/documents/2015/UNAIDS_PCB37_15-18 [Accessed 15 August 2017].

32. ***United Nations, 2016. The Millennium Development Goals Report 2015, New York.

33. ***World Health Organization, 2015. The H4+ partnership joint support to improve women's and children's health: progress report 2014. Available at: http://www.who.int/iris/bitstream/10665/134746/1/WHO_RHR_14.27_eng.pdf [Accessed 10 May 2016].

34. *** World Health Organization, 2008. Taskshifting: Global Recommendations and Guidelines. Available at: http://www.who.int/healthsystems/TTR-TaskShifting.pdf [Accessed 15 December 2015]. 